\title{
Diurnal Change of Plasma Insulin, Free Fatty Acids and Human Growth Hormone in Diabetics during Bonito Insulin Treatment
}

\author{
Takayoshi Toyota*, Shin-Ichiro Sato*, Akira Ohneda \\ and Yoshio Goto* \\ The Third Department of Internal Medicine, $\dagger$ Tohoku \\ University School of Medicine, Sendai
}

\begin{abstract}
Toyota, T., Sato, S., OhNeda, A. and Goto, Y. Diurnal Change of Plasma Insulin, Free Fatty Acids and Human Growth Hormone in Diabetics during Bonito Insulin Treatment. Tohoku J. exp. Med., 1972, 107 (2), 167-172 The present study was undertaken to see whether the function of the pancreas in diabetics is improved by the exogenous insulin treatment. In seven diabetic patients, bonito insulin was injected every day until the urine sugar became negative, and the diurnal change of plasma insulin was estimated before insulin injection and during the urine sugar-free period. Plasma insulin area, calculated by our formula, increased in six of the patients. As bonito insulin does not show any immunological cross-reaction with pork, beef and human insulin, this increase in plasma insulin area after the bonito insulin treatment may be due to an improvement of the impaired function of the pancreas — bonito insulin; insulin treatmen1; plasma insulin; diurnal rhythm
\end{abstract}

The radioimmunoassay technique (Yalow and Berson 1960) has enriched our knowledge on blood insulin levels in various conditions and also has given many evidences to support the classical theories on the pathogenesis of diabetes mellitus. In the previous study (Toyota 1969), we reported the diurnal fluctuation of plasma insulin before and after the sulfonylurea treatment and found that the plasma insulin levels of the mild diabetics decreased after the control of blood sugar, and on the contrary, the plasma insulin levels of the moderately severe diabetics increased by the treatment. These observations impelled us to extend our study to see a change of plasma insulin level after the treatment with insulin. The estimation of the endogenous circulating insulin during insulin treatment is possible if bonito insulin is used, because it has no immunological cross-reaction ith pork, beef or human insulin (Yamagata et al. 1969).

\section{Materials and Methods}

Seven primary diabetic inpatients were selected for this study. Age of the cases ranged from 36 to 52 years old. Five of them were male and two female (Table 1). The

Received for publication, September 29, 1971.

* Present address: The Third Department of Internal Medicine, Hirosaki University School of Medicine, Zaifucho 5, Hirosaki, Japan.

$\dagger$ Director: Prof. S. Yamagata. 
diet of $1,600 \mathrm{kcal}$ or $1,800 \mathrm{kcal}$ was given to the patients during the study. Breakfast was served at 8:00 a.m., lunch at noon and supper at 4:30 p.m. No other foods were given during this experiment. Blood samples for blood glucose, plasma insulin, free fatty acids (FFA) and growth hormone (GH) analyses were obtained by antecubital venipuncture with a heparinized syringe at 7:00 a.m., 11:00 a.m., 1:00 p.m., 4:00 p.m., 6:00 p.m., 12:00 midnight and 4:00 a.m. and 7:00 a.m. of the following morning. Daily excretion of urine sugar was measured during the hospitalization. The initial determination of diurnal rhythm of blood glucose, plasma insulin, FFA and GH was performed before the start of the bonito insulin therapy and the second determination was made after 8 to 33 days of the therapy, when the 24-hour urinary sugar excretion became zero. Unmodified bonito insulin was injected subcutaneously thirty minutes before each meal, i.e. at 7:30, at 11:30 a.m. and 4:30 p.m. Total dosage of bonito insulin ranged 294 to 1,144 units in the series (Table 1).

Blood glucose was determined by the glucose oxidase method of Teller (1956). The plasma insulin was extracted by the modified method of Jephcott (1931) and was measured by the double antibody method of Morgan and Lazarow (1962). FFA was estimated by the method of Itaya-Ui (1965) and GH was measured by double antibody method of Utiger (1964). Antibody to human growth hormone and ${ }^{131} \mathrm{I}-\mathrm{GH}$ used here were prepared by Dainabot Radioisotope Laboratory. Bonito insulin was supplied by Shimizu Pharmaceutical Co.

The area of blood glucose, plasma insulin, FFA and GH were calculated by the formula described in the previous paper (Toyota 1969).

TABLE 1. Seven diabetic patients and dosage of bonito insulin

\begin{tabular}{l|c|c|c|c|c|c|c|c}
\hline No. & Sex & Age & $\begin{array}{c}\text { Height } \\
\text { (cm) }\end{array}$ & $\begin{array}{c}\text { Weight } \\
\text { (kg) }\end{array}$ & \multicolumn{2}{|c|}{$\begin{array}{c}\text { Daily excretion of } \\
\text { urine sugar (g/day) }\end{array}$} & $\begin{array}{c}\text { Bonito } \\
\text { insulin } \\
\text { (units) }\end{array}$ & $\begin{array}{c}\text { Duration } \\
\text { of } \\
\text { tratment } \\
\text { (days) }\end{array}$ \\
\hline S.T. & M & 52 & 154 & 60 & 20 & 0 & 948 & 33 \\
H.T. & F & 34 & 160 & 44 & 42 & 0 & 1144 & 26 \\
S.C. & M & 43 & 160 & 53 & 135 & 0 & 480 & 8 \\
S.I. & F & 54 & 152 & 50 & 34 & 0 & 670 & 16 \\
N.S. & M & 36 & 160 & 47 & 65 & 0 & 550 & 22 \\
S.K. & M & 40 & 168 & 77 & 64 & 0 & 360 & 12 \\
E.S. & M & 37 & 166 & 53 & 16 & 0 & 294 & 16
\end{tabular}

\section{Results}

The results are shown in Tables 2 and 3. The blood glucose levels decreased significantly after the bonito insulin treatment in all patients. This was clearly shown in the changes of blood glucose area; namely, it decreased from $6,020 \pm 535$ to $2,666 \pm 236 \mathrm{mg} / 100 \mathrm{ml} / 24 \mathrm{hrs}(\mathrm{P}<0.01)$. The plasma insulin area, however, increased in six of the seven patients and decreased in one case after the treatment. The mean value of the insulin area changed from $794 \pm 129$ to $1,008 \pm 249 \mu \mathrm{U} / \mathrm{ml}$ $124 \mathrm{hrs}$ after the bonito insulin therapy $(0.1<\mathrm{P}<0.2)$.

The insulin area of Case 6 alone, in which the initial fasting plasma insulin was abnormally high, decreased from 1,353 to $963 \mu \mathrm{U} / \mathrm{ml} / 24$ hrs by the treatment (Table 3). This result suggested that the endogenous insulin secretion increased when the blood glucose closed to normal range by the insulin therapy, unless the initial plasma insulin level was abnormally high.

Case 3 was an insulin-deficient diabetic and his plasma insulin area was $310 \mu \mathrm{U} /$ 
$\mathrm{ml} / 24 \mathrm{hrs}$ and the blood glucose area was $8,956 \mathrm{mg} / 100 \mathrm{ml} / 24 \mathrm{hrs}$ (mean value of normal plasma insulin area and blood glucose area are $628 \mu \mathrm{U} / \mathrm{ml} / 24 \mathrm{hrs}$ and 1,746 $\mathrm{mg} / 100 \mathrm{ml} / 24 \mathrm{hrs}$, respectively). After injection of 480 units of bonito insulin for 8 days, his insulin area increased and the blood glucose area decreased. Case 2 was an moderately severe diabetic. His plasma insulin area was $615 \mu \mathrm{U} / \mathrm{ml} / 24$ hrs before the treatment. In this case 1,144 units of bonito insulin was used for 25 days until the urinary sugar disappeared. These results indicated that the sensitivity to the insulin was variable among the patients (Table 1).

FFA and GH levels became within the normal range after the bonito insulin therapy (Table 2); namely, FFA area changed from $14.78 \pm 4.4$ to $9.27 \pm 1.7$ $\mathrm{mEq} / \mathrm{L} / 24 \mathrm{hrs}$ by the insulin treatment $(0.1<\mathrm{P}<0.2)$, and growth hormone area changed from $128 \pm 46$ to $99.5 \pm 27 \mathrm{~m} \mu \mathrm{g} / \mathrm{ml} / 24 \mathrm{hrs}$ after the bonito insulin treatment $(0.1<\mathrm{P}<0.2)$.

\section{Discussion}

It is reasonable to expect that the plasma insulin levels may become normal when the blood glucose fluctuates within nearly normal range with the management of diabetes, unless the insulin antagonists still remain and the islet-cells of the pancreas are destroyed.

Lukens and Dohan (1942) found that the hydropic-degenerated $\beta$-cells of the experimental diabetic cat pancreas showed a regeneration, when the hyperglycemic condition was managed with insulin. On the basis of this experiment, it was surmised that the plasma insulin level of diabetes increased after the exogenous insulin treatment because of the amelioration of islet-cells function. It is easy to analogize this experiment in clinical phenomenon, i.e. the remission of diabetic state observed in juvenile diabetics.

Our result showed that the plasma insulin area in six of seven patients increased after the bonito insulin treatment. This suggests that the injection of bonito insulin improves the function of pancreatic islet-cells.

On the other hand, two different results are reported concerning the effect of the exogenous insulin on the insulin secretion from the pancreas. Grodsky and his co-workers (1968) found no suppressive effect of the bonito insulin on the insulin secretion from rat pancreas. However, an inhibition of the insulin release from the pancreas by insulin in vitro was demonstrated by Sodoyez et al. (1969), Hahn and Michael (1970), and Iversen (1971). Since in our study the plasma insulin area increased by the treatment with bonito insulin, it seems unlikely that bonito insulin suppresses the secretion of insulin from the pancreas.

The previous study (Toyota 1969) showed that the insulin area of the mild diabetic decreased significantly with the sulfonylurea treatment and we speculated that it was due to an increase of the sensitivity of the tissues to insulin. If the insulin antagonists are present in diabetics, the insulin may be secreted in a large amount from the pancreas. FFA and $\mathrm{GH}$, which are thought to be insulin antagonists, did not increase after the bonito insulin therapy in this study. 
TABLE 2. Mean value of diurnal change of blood glucose, plasma

\begin{tabular}{|c|c|c|c|c|c|}
\hline & & $\begin{array}{c}7 \\
\text { a.m. }\end{array}$ & $\begin{array}{c}9 \\
\text { a.m. }\end{array}$ & $\begin{array}{c}11 \\
\text { a.m. }\end{array}$ & $\begin{array}{c}1 \\
\text { p.m. }\end{array}$ \\
\hline \multirow{2}{*}{$\begin{array}{l}\text { Blood } \\
\text { glucose } \\
\mathrm{mg} / 100 \mathrm{ml}\end{array}$} & Before & $\begin{array}{r}185.0 \\
\pm 12.2\end{array}$ & $\begin{array}{r}308.9 \\
+21.4\end{array}$ & $\begin{array}{r}276.2 \\
\pm 31.0\end{array}$ & $\begin{array}{r}320.9 \\
\pm 40.3\end{array}$ \\
\hline & After & $\begin{array}{l}105.1 \\
\pm 5.4\end{array}$ & $\begin{array}{l}138.7 \\
\pm 6.7\end{array}$ & $\begin{array}{l}107.1 \\
\pm 9.4\end{array}$ & $\begin{array}{r}91.3 \\
\pm 15.9\end{array}$ \\
\hline \multirow{2}{*}{$\begin{array}{c}\text { Plasma } \\
\text { insulin } \\
\mu \mathrm{U} / \mathrm{ml}\end{array}$} & Before & $\begin{array}{r}26.1 \\
\pm 5.5\end{array}$ & $\begin{array}{r}34.0 \\
\pm 5.8\end{array}$ & $\begin{array}{r}38.3 \\
\pm 6.2\end{array}$ & $\begin{array}{r}35.9 \\
\pm 2.7\end{array}$ \\
\hline & After & $\begin{array}{r}42.4 \\
\pm 8.6\end{array}$ & $\begin{array}{r}48.9 \\
\pm 12.6\end{array}$ & $\begin{array}{r}51.4 \\
\pm 11.6\end{array}$ & $\begin{array}{r}49.7 \\
\pm 13.6\end{array}$ \\
\hline \multirow[t]{2}{*}{$\begin{array}{l}\text { FFA } \\
\mathrm{mEq} / \mathrm{L}\end{array}$} & Before & $\begin{array}{r}0.62 \\
\pm 0.09\end{array}$ & $\begin{array}{r}0.51 \\
\pm 0.16\end{array}$ & $\begin{array}{r}0.39 \\
\pm 0.08\end{array}$ & $\begin{array}{r}0.48 \\
\pm 0.11\end{array}$ \\
\hline & After & $\begin{array}{r}0.58 \\
\pm 0.06\end{array}$ & $\begin{array}{r}0.45 \\
\pm 0.11\end{array}$ & $\begin{array}{r}0.31 \\
\pm 0.05\end{array}$ & $\begin{array}{r}0.32 \\
\pm 0.08\end{array}$ \\
\hline \multirow[t]{2}{*}{$\begin{array}{l}\mathrm{HGH} \\
\mathrm{m} \mu \mathrm{g} / \mathrm{ml}\end{array}$} & Before & $\begin{array}{r}4.1 \\
\pm 0.7\end{array}$ & $\begin{array}{r}3.7 \\
\pm 1.8\end{array}$ & $\begin{array}{r}6.2 \\
\pm 2.2\end{array}$ & $\begin{array}{r}7.7 \\
\pm 5.1\end{array}$ \\
\hline & After & $\begin{array}{l}4.0 \\
\pm 0.43\end{array}$ & $\begin{array}{r}2.3 \\
\pm 0.5\end{array}$ & $\begin{array}{r}4.4 \\
\pm 1.3\end{array}$ & $\begin{array}{r}3.7 \\
\pm 1.4\end{array}$ \\
\hline
\end{tabular}

Mean \pm S.E. $\quad \mathrm{N}=7$

TABLE 3. Diurnal change of blood glucose and plasma insulin

\begin{tabular}{|c|c|c|c|c|c|c|c|c|c|c|c|}
\hline \multirow{3}{*}{ Case } & & \multicolumn{9}{|c|}{ Time } & \multirow{3}{*}{$\begin{array}{c}\text { Blood } \\
\text { glucose } \\
\text { area } \\
(\mathrm{mg} / 100 \mathrm{ml} / \\
24 \text { hours })\end{array}$} \\
\hline & & $\begin{array}{c}7 \\
\text { a.m. }\end{array}$ & $\begin{array}{c}9 \\
\text { a.m. }\end{array}$ & $\begin{array}{c}11 \\
\text { a.m. }\end{array}$ & $\begin{array}{c}1 \\
\text { p.m. }\end{array}$ & $\begin{array}{c}4 \\
\text { p.m. }\end{array}$ & $\begin{array}{c}6 \\
\text { p.m. }\end{array}$ & $\begin{array}{c}\text { Midnight } \\
0\end{array}$ & $\begin{array}{c}4 \\
\text { a.m. }\end{array}$ & $\begin{array}{c}7 \\
\text { a.m. }\end{array}$ & \\
\hline & & \multicolumn{9}{|c|}{ Blood glucose $(\mathrm{mg} / 100 \mathrm{ml})$} & \\
\hline \multirow{2}{*}{1} & Before & 238 & 238 & 230 & 240 & 209 & 266 & 254 & 209 & 236 & 5,713 \\
\hline & After & 116 & 180 & 139 & 103 & 153 & 109 & 98 & 94 & 116 & 2,764 \\
\hline \multirow{2}{*}{2} & Before & 222 & 316 & 276 & 273 & 247 & 305 & 242 & 216 & 195 & 6,143 \\
\hline & After & 152 & 202 & 161 & 164 & 98 & 121 & 151 & 130 & 141 & 3,435 \\
\hline \multirow{2}{*}{3} & Before & 201 & 417 & 438 & 556 & 396 & 443 & 333 & 236 & 215 & 8,956 \\
\hline & After & 94 & 124 & 89 & 64 & 83 & 44 & 60 & 80 & 89 & 1,729 \\
\hline \multirow{2}{*}{4} & Before & 173 & 322 & 327 & 283 & 301 & 494 & 238 & 169 & 154 & 6,797 \\
\hline & After & 126 & 130 & 151 & 65 & 126 & 89 & 122 & 110 & 119 & 2,673 \\
\hline \multirow{2}{*}{5} & Before & 164 & 258 & 257 & 360 & 202 & 263 & 232 & 197 & 209 & 5,864 \\
\hline & After & 89 & 132 & 100 & 185 & 141 & 189 & 156 & 119 & 146 & 3,550 \\
\hline \multirow{2}{*}{6} & Before & 152 & 268 & 254 & 210 & 173 & 201 & 136 & 121 & 152 & 4,253 \\
\hline & After & 115 & 135 & 99 & 85 & 146 & 131 & 84 & 103 & 76 & 2,536 \\
\hline \multirow{2}{*}{7} & Before & 151 & 343 & 152 & 324 & 239 & 266 & 159 & 104 & 108 & 4,778 \\
\hline & After & 87 & 128 & 82 & 91 & 69 & 180 & 60 & 75 & 78 & 1,977 \\
\hline
\end{tabular}


insulin, FFA and GH after bonito insulin treatment

\begin{tabular}{|c|c|c|c|c|c|}
\hline \multicolumn{2}{|c|}{ Time } & \multirow[b]{2}{*}{$\begin{array}{l}12 \\
\text { midnight }\end{array}$} & \multirow[b]{2}{*}{$\begin{array}{c}4 \\
\text { a.m. }\end{array}$} & \multirow[b]{2}{*}{$\begin{array}{c}7 \\
\text { a.m. }\end{array}$} & \multirow[b]{2}{*}{ Area } \\
\hline $\begin{array}{c}4 \\
\text { p.m. }\end{array}$ & $\begin{array}{c}6 \\
\text { p.m. }\end{array}$ & & & & \\
\hline $\begin{array}{r}265.7 \\
\pm 25.2\end{array}$ & $\begin{array}{r}319.7 \\
\pm 37.5\end{array}$ & $\begin{array}{r}227.7 \\
\pm 22.7\end{array}$ & $\begin{array}{r}178.9 \\
\pm 17.5\end{array}$ & $\begin{array}{r}181.3 \\
\pm 15.7\end{array}$ & $\begin{array}{r}6020 \\
\pm 535\end{array}$ \\
\hline $\begin{array}{r}113.1 \\
\pm 12.8\end{array}$ & $\begin{array}{r}104.0 \\
\pm 16.9\end{array}$ & $\begin{array}{r}94.3 \\
\pm 12.2\end{array}$ & $\begin{array}{r}94.6 \\
\pm 5.9\end{array}$ & $\begin{array}{l}103.1 \\
\pm 8.9\end{array}$ & $\begin{array}{r}2666 \\
\pm 263\end{array}$ \\
\hline $\begin{array}{r}35.9 \\
\pm 2.8\end{array}$ & $\begin{array}{r}37.1 \\
\pm 6.4\end{array}$ & $\begin{array}{r}31.9 \\
\pm 6.3\end{array}$ & $\begin{array}{r}28.1 \\
\pm 4.2\end{array}$ & $\begin{array}{r}25.0 \\
\pm 4.7\end{array}$ & $\begin{array}{r}794 \\
\pm 129\end{array}$ \\
\hline $\begin{array}{r}39.4 \\
\pm 5.5\end{array}$ & $\begin{array}{r}44.6 \\
\pm 10.2\end{array}$ & $\begin{array}{r}38.0 \\
\pm 5.7\end{array}$ & $\begin{array}{r}43.1 \\
\pm 10.3\end{array}$ & $\begin{array}{r}38.9 \\
\pm 8.7\end{array}$ & $\begin{array}{r}1008 \\
\pm 249\end{array}$ \\
\hline $\begin{array}{r}0.48 \\
\pm .09\end{array}$ & $\begin{array}{r}0.46 \\
\pm 0.09\end{array}$ & $\begin{array}{r}0.54 \\
\pm 0.09\end{array}$ & $\begin{array}{r}0.51 \\
\pm 0.10\end{array}$ & $\begin{array}{r}0.65 \\
\pm 0.13\end{array}$ & $\begin{array}{l}14.78 \\
\pm 4.4\end{array}$ \\
\hline $\begin{array}{r}0.34 \\
\pm 0.08\end{array}$ & $\begin{array}{r}0.34 \\
\pm 0.10\end{array}$ & $\begin{array}{r}0.36 \\
\pm 0.09\end{array}$ & $\begin{array}{r}0.52 \\
\pm 0.09\end{array}$ & $\begin{array}{r}0.55 \\
\pm 0.08\end{array}$ & $\begin{array}{r}9.27 \\
\pm 1.7\end{array}$ \\
\hline $\begin{array}{r}3.4 \\
\pm 1.0\end{array}$ & $\begin{array}{r}5.2 \\
\pm 1.7\end{array}$ & $\begin{array}{r}8.3 \\
\pm 2.9\end{array}$ & $\begin{array}{r}4.3 \\
\pm 2.2\end{array}$ & $\begin{array}{r}2.7 \\
\pm 0.5\end{array}$ & $\begin{array}{l}128.1 \\
\pm 460\end{array}$ \\
\hline $\begin{array}{r}3.7 \\
\pm 0.8\end{array}$ & $\begin{array}{r}3.1 \\
\pm 0.9\end{array}$ & $\begin{array}{r}7.1 \\
\pm 2.2\end{array}$ & $\begin{array}{r}3.3 \\
\pm 0.7\end{array}$ & $\begin{array}{r}7.0 \\
\pm 3.7\end{array}$ & $\begin{array}{r}99.5 \\
\pm 27.1\end{array}$ \\
\hline
\end{tabular}

in seven diabetic patients after bonito insulin treatment

\begin{tabular}{|c|c|c|c|c|c|c|c|c|c|}
\hline \multicolumn{9}{|c|}{ Time } & \multirow{3}{*}{$\begin{array}{c}\text { Insulin } \\
\text { Area } \\
(\mu \mathrm{U} / \mathrm{ml} / \\
24 \text { hours })\end{array}$} \\
\hline $\begin{array}{c}7 \\
\text { a.m. }\end{array}$ & $\begin{array}{c}9 \\
\text { a.m. }\end{array}$ & $\begin{array}{c}11 \\
\text { a.m. }\end{array}$ & $\begin{array}{c}1 \\
\text { p.m. }\end{array}$ & $\begin{array}{c}4 \\
\text { p.m. }\end{array}$ & $\begin{array}{c}6 \\
\text { p.m. }\end{array}$ & $\begin{array}{c}\text { Midnight } \\
0\end{array}$ & $\begin{array}{c}4 \\
\text { a.m. }\end{array}$ & $\begin{array}{c}7 \\
\text { a.m. }\end{array}$ & \\
\hline \multicolumn{9}{|c|}{ Plasma insulin $(\mu \mathrm{U} / \mathrm{ml})$} & \\
\hline $\begin{array}{l}10 \\
38\end{array}$ & $\begin{array}{r}32 \\
8\end{array}$ & $\begin{array}{l}14 \\
30\end{array}$ & $\begin{array}{l}28 \\
26\end{array}$ & $\begin{array}{l}20 \\
34\end{array}$ & $\begin{array}{l}32 \\
44\end{array}$ & $\begin{array}{l}26 \\
40\end{array}$ & $\begin{array}{l}26 \\
26\end{array}$ & $\begin{array}{r}8 \\
20\end{array}$ & $\begin{array}{l}572 \\
781\end{array}$ \\
\hline $\begin{array}{l}16 \\
10\end{array}$ & 28 & 58 & $\begin{array}{l}38 \\
40\end{array}$ & $\begin{array}{l}20 \\
18\end{array}$ & $\begin{array}{l}18 \\
30\end{array}$ & $\begin{array}{l}18 \\
20\end{array}$ & $\begin{array}{l}20 \\
20\end{array}$ & $\begin{array}{l}20 \\
22\end{array}$ & $\begin{array}{l}615 \\
957\end{array}$ \\
\hline $\begin{array}{r}14 \\
8\end{array}$ & $\begin{array}{l}10 \\
24\end{array}$ & $\begin{array}{l}16 \\
24\end{array}$ & $\begin{array}{l}24 \\
18\end{array}$ & $\begin{array}{l}10 \\
16\end{array}$ & $\begin{array}{l}14 \\
18\end{array}$ & $\begin{array}{r}10 \\
8\end{array}$ & $\begin{array}{l}8 \\
2\end{array}$ & $\begin{array}{l}10 \\
10\end{array}$ & $\begin{array}{l}310 \\
320\end{array}$ \\
\hline $\begin{array}{l}26 \\
90\end{array}$ & $\begin{array}{r}58 \\
110\end{array}$ & $\begin{array}{l}50 \\
68\end{array}$ & $\begin{array}{l}38 \\
38\end{array}$ & $\begin{array}{l}56 \\
38\end{array}$ & $\begin{array}{l}46 \\
38\end{array}$ & $\begin{array}{l}44 \\
56\end{array}$ & $\begin{array}{l}40 \\
58\end{array}$ & $\begin{array}{l}38 \\
52\end{array}$ & $\begin{array}{l}1,064 \\
1,292\end{array}$ \\
\hline $\begin{array}{l}35 \\
35\end{array}$ & $\begin{array}{l}28 \\
38\end{array}$ & $\begin{array}{l}40 \\
38\end{array}$ & $\begin{array}{l}35 \\
38\end{array}$ & $\begin{array}{l}38 \\
28\end{array}$ & $\begin{array}{l}40 \\
32\end{array}$ & $\begin{array}{l}26 \\
28\end{array}$ & $\begin{array}{l}26 \\
96\end{array}$ & $\begin{array}{l}26 \\
82\end{array}$ & $\begin{array}{r}783 \\
1,049\end{array}$ \\
\hline $\begin{array}{l}56 \\
40\end{array}$ & $\begin{array}{l}54 \\
30\end{array}$ & $\begin{array}{l}54 \\
42\end{array}$ & $\begin{array}{l}48 \\
56\end{array}$ & $\begin{array}{l}66 \\
60\end{array}$ & $\begin{array}{l}68 \\
36\end{array}$ & $\begin{array}{l}64 \\
36\end{array}$ & $\begin{array}{l}42 \\
34\end{array}$ & $\begin{array}{l}40 \\
18\end{array}$ & $\begin{array}{r}1,353 \\
963\end{array}$ \\
\hline $\begin{array}{l}26 \\
40\end{array}$ & $\begin{array}{l}28 \\
60\end{array}$ & $\begin{array}{l}36 \\
82\end{array}$ & $\begin{array}{l}40 \\
82\end{array}$ & $\begin{array}{l}36 \\
82\end{array}$ & $\begin{array}{r}42 \\
124\end{array}$ & $\begin{array}{l}35 \\
40\end{array}$ & $\begin{array}{l}35 \\
52\end{array}$ & $\begin{array}{l}28 \\
42\end{array}$ & $\begin{array}{r}861 \\
1,697\end{array}$ \\
\hline
\end{tabular}


Therefore, the increase of the plasma insulin area after the treatment is probably due to an improvement of the islet function and not related with an increase of antagonist in the plasma.

In conclusion, the dysfunction of the islet cells of the pancreas in diabetics is improved by the bonito insulin therapy. Further investigation is necessary to see the effect of a long-term insulin therapy on the endogenous insulin secretion of the pancreas.

\section{References}

1) Grodsky, G.M., Curry, D.L., Benett, L.L. \& Rodrigo, J.J. (1968) Factors influencing different rates of insulin release in vitro. Acta diab. latina, 2 (Suppl. 1), 140-161.

2) Hahn, H.J. \& Michael, R. (1970) Inhibition of insulin release by endogenous insulin in vitro. Horm. Metab. Res. 2, 119-120.

3) Itaya, K. \& Ui, M. (1965) Colorimetric determination of free fatty acids in biological fluids. J. Lipid Res., 6, 16-20.

4) Iversen. J. \& Miles, D.W. (1971) Evidence for a feedback inhibition of insulin on insulin secretion in the isolated, perfused canine pancreas. Dixbetes, 20, 1-9.

5) Jephcott, C.M. (1931) Extraction of insulin and stability of various preparations. Trans. roy. Soc. Canada, Sect. V, 25, 183-186.

6) Lukens, F.D.W. \& Dohan, F.C. (1942) Pituitary diabetes in the cat: Recovery following insulin or dietary treatment. Endocrinology, 30, 175-202.

7) Morgan, C.R. \& Lazarow, A. (1962) Immunoassay of insulin using a two-antibody system. Proc. Soc. exp. Biol. Med., 110, 29-32.

8) Sodoyez, J.-C., Sodoyez-Goffaux, F. \& Foa, P.P. (1969) Evidence for an insulininduced inhibition of insulin release by isolated islets of Langerhans. Proc. Soc. exp. Biol. Med., 130, 568-571.

9) Teller, J.D. (1956) Direct, quantitative, colorimetric determination of serum or plasma glucose. Abstract of papers, the 130th meeting, Amer. Chem. Soc., Atlantic City N.J. Sept.

10) Toyota, T. (1969) Diurnal change of plasma insulin in diabetics with sulfonylurea treatment. Tohoku J. exp. Med., 98, 345-356.

11) Utiger, R.D. (1964) Extraction and radioimmunoassay of growth hormone in human serum. J. clin. Endocrinol., 24, 60-67.

12) Yalow, R.S. \& Berson, S.A. (1960) Immunoassay of endogenous plasma insulin in man. J. clin. Invest., 39, $1157-1175$.

13) Yamagata, S., Goto, Y., Ohneda, A., Toyota, T \& Sato, S. (1969) Diurnal change of plasma insulin in diabetics by the treatment with exogenous insulin. Folia endocrinol. japonica (Jap.), 45, 475-476. 